DOI: https://doi.org/10.11144/Javeriana.upsy16-2.alea

\title{
Adolescentes latinoamericanos en España: Aculturación, autonomía conductual, conflictos familiares y bienestar subjetivo*
}

\section{Latin American adolescents in Spain: Acculturation, behavioral autonomy, family conflicts and subjective well-being}

Recepción: 22 Abril 2014 | Aprobación: 24 Enero 2017

\author{
LAURA LARA ${ }^{a}$ \\ Universidad Autónoma de Chile, Chile \\ ORCID: http://orcid.org/0000-0001-6420-2398
}

a Autora de correspondencia. Correo electrónico: llarav@uautonoma.cl

Para citar este artículo: Lara, L. (2017) Adolescentes latinoamericanos en España: Aculturación, autonomía conductual, conflictos familiares y bienestar subjetivo. Universitas Psychologica 16(2), 1-11. https://doi.org/10.11144/Javeriana.upsy16-2. alea

\section{RESUMEN}

En este estudio se analizan los perfiles de aculturación de 331 adolescentes latinoamericanos residentes en España y su relación con sus niveles de autonomía conductual, los conflictos que mantienen con sus progenitores y su bienestar subjetivo. Los resultados de los análisis de conglomerados confirman la existencia de cuatro perfiles de aculturación: integración $(\mathrm{n}=113)$, separación $(\mathrm{n}=100)$, marginación $(\mathrm{n}=63)$ y asimilación $(\mathrm{n}=55)$. Respecto a las diferencias entre perfiles en las variables estudiadas, los resultados de los análisis de la varianza mostraron que no existieron diferencias estadísticamente significativas en la autonomía conductual ni en la frecuencia de conflictos; pero sí en el bienestar subjetivo, siendo el perfil de marginación fue el que presentó una menor satisfacción vital.

Palabras clave

aculturación; adolescentes; autonomía; conflictos; satisfacción vital

\section{ABSTRACT}

In this study, we analyze the acculturation profiles of 331 Latin-American adolescents living in Spain, and their relation with their levels of behavioral autonomy, conflicts with their parents, and their subjective well-being. Results confirm the existence of four acculturation strategies: integration $(\mathrm{n}=113)$, separation $(\mathrm{n}=100)$, marginalization $(\mathrm{n}=63)$, and assimilation $(\mathrm{n}=55)$. Regarding differences between profiles, results from analyses of variance showed there were not statistical differences in adolescents' behavioral autonomy, nor in the frequency of parentadolescent conflict; there was in subjective well-being, being the marginalization profile the one that presenting less life satisfaction.

Keywords

acculturation; adolescents; autonomy; conflicts; life satisfaction

\section{Introducción}

Actualmente ninguna investigación que pretenda abordar el estudio del contacto entre diferentes grupos culturales puede pasar por alto la consideración del modelo bidimensional de aculturación desarrollado por Berry (1997). Este modelo plantea que para realizar una adaptación exitosa a la nueva 
sociedad, las personas que emigran no tienen que abandonar sus culturas de origen, tal como lo establecían las primeras aproximaciones al estudio de la aculturación, que incluso consideraban perjudicial que se mantuviesen los valores y costumbres de origen (e.g., Horrocks, 1962). De esta forma, se establecen cuatro estrategias de aculturación (asimilación, separación, integración y marginación) en función de dos dimensiones independientes claves, la orientación hacia la cultura de origen y hacia la nueva cultura. La estrategia de asimilación se produce cuando los individuos no desean mantener su herencia cultural y buscan interacciones diarias con otras culturas, mientras que la situación contraria da lugar a la separación. Cuando los individuos están interesados tanto en mantener la cultura de origen como en llevar a cabo interacciones con los miembros de la sociedad mayoritaria, se produciría la estrategia de integración. Por último, la marginación ocurre cuando hay pocas posibilidades o interés en el mantenimiento de la cultura (a menudo debido a una pérdida cultural forzada) y poco interés en mantener relaciones con los miembros de la cultura mayoritaria (a menudo por razones de exclusión o discriminación).

Los estudios internacionales ponen en relieve, de forma general, que la estrategia de integración es la que se presenta en mayor medida, tanto en muestras de adultos como de adolescentes, y es la que mejores resultados tiene para la adaptación de los inmigrantes (Berry, Phinney, Sam, \& Vedder, 2006; Coatsworth, MaldonadoMolina, Pantin, \& Szapocznik, 2005; David, Okazaki, \& Saw, 2009; Nguyen \& BenetMartínez, 2013; Shafaei, Razak, \& Nejati, 2016). Parece existir consenso en considerar positivo para el desarrollo, tanto el contacto con la nueva cultura como el mantenimiento de la de origen. De hecho, los modelos centrados en el desarrollo de los adolescentes inmigrantes conciben la aculturación en términos positivos, como un proceso de desarrollo destinado a ganar competencia dentro de más de un contexto sociocultural (Sam \& Oppedal, 2003). La idea que sustenta esta relación es que fuertes identidades nacionales o étnicas proporcionan un sentido de estabilidad emocional y seguridad personal, y pueden ser considerados recursos para el bienestar y la adaptación (Vedder, Van de Vijver, \& Liebkind, 2006). Además, mantener identidades biculturales es la opción más beneficiosa para el desarrollo de los menores, convirtiéndose en una ventaja debido a que les permite desenvolverse en entornos multiétnicos y multiculturales (Trueba, 2001), y ha sido asociada de forma consistente con un mejor ajuste psicológico y sociocultural (véase el metaanálisis realizado por Nguyen \& Benet-Martínez, 2013).

Esta misma relación se confirma en los estudios realizados en España (Briones, 2010), y específicamente con adolescentes latinoamericanos (Matsunaga, Hecht, Elek, \& Ndiaye, 2010; Sobral, Gómez-Fraguela, Luengo, Romero, \& Villar, 2010). En todas las investigaciones consultadas, la estrategia de marginación fue la menos predominante, siendo además la orientación que peores consecuencias tiene, dado que deja a los jóvenes sin identificación con los valores y las normas de ninguna cultura, provocándoles una falta de identidad, un sentir que no se pertenece a ninguna cultura. Sin embargo, las investigaciones se han centrado de forma generalizada en las consecuencias negativas del proceso de aculturación, como por ejemplo la conducta antisocial (Sobral, Gómez-Fraguela, Romero, Luengo, \& Villar, 2012), y han dejado de lado aspectos tan importantes como el bienestar subjetivo, motivo por el cual focalizamos en la satisfacción vital en el presente estudio.

Un aspecto importante, y que ha sido menos estudiado, es cómo influyen estas estrategias de aculturación en las relaciones familiares de los adolescentes, en temas tan cruciales como su autonomía conductual o los conflictos que mantienen con sus progenitores. En el desarrollo de las relaciones entre los adolescentes inmigrantes y sus progenitores intervienen muchos factores. La revisión de las investigaciones disponibles muestra la existencia de posiciones y resultados contrapuestos. La cantidad de medidas desarrolladas, así como la utilización de los indicadores de aproximación 
(como el uso del lenguaje o los años de residencia en el nuevo país), en lugar de servir como medida directa de la aculturación, dificultan estas comparaciones. Además, en los estudios realizados se entremezclan con frecuencia las concepciones de la cultura con el estatus de inmigrante, al considerar no solo a los jóvenes que han realizado la emigración, sino a los que ya han nacido en el país de acogida, e incluso a las terceras generaciones. Para evitar este inconveniente, en esta investigación nos centramos en adolescentes de primera generación, controlando que ambos progenitores también lo sean y procedan del mismo país. De este modo, se consolida como uno de los primeros estudios realizados en España que focaliza específicamente en esta población. Sin lugar a dudas, son muchos los factores que pueden influir sobre el curso de estas relaciones en las familias que emigran. También son muchas las variables que influyen en las relaciones que tienen las familias que no han emigrado, pero a estas hay que añadirles el tener que adaptarse a una nueva cultura cuando los menores aún no han terminado el proceso de endoculturación en sus culturas de origen familiares. Los padres y madres transmiten a sus hijos e hijas los valores de su cultura que consideran importantes para su adecuado desarrollo en la sociedad, pero puede que no sean los adecuados en el nuevo contexto. De esta forma, en medio de este proceso, todo el esquema puede tener que volver a reestructurarse para conseguir su correcto funcionamiento como miembros de la sociedad.

Entre los factores que pueden influir en las relaciones entre progenitores e hijos e hijas, a menudo se ha citado la diferencia inicial entre las culturas que entran en contacto, en donde son más armoniosas las relaciones cuando esta distancia es menor (Ward \& Geeraert, 2016). Por ejemplo, Rudmin (2003) señala que la facilidad para integrar la cultura de origen con la de acogida dependerá en gran medida del grado de similitud entre ellas. A este respecto, el lenguaje ha sido uno de los aspectos más referenciados. Por ejemplo, en Estados Unidos tendrá más facilidad de adaptación una persona que proceda de un país en el que se hable inglés (Schwartz, Unger,
Zamboanga, Byron, \& Szapocznik, 2010), de la misma forma que en España la tendrá una persona que procede de un país en el que se hable castellano. Pero también han sido señalados otros aspectos relacionados con los valores predominantes en las culturas (Kashima \& AbuRayya, 2014). Frecuentemente se compara entre sociedades colectivistas e individualistas, debido a que la mayoría de las investigaciones se han realizado en países considerados individualistas en contraposición a los países de los que proceden los inmigrantes, usualmente calificados como colectivistas. Por ejemplo, Choi, He y Barachi (2008) sugieren que el choque cultural puede ser más importante en las familias asiáticas en Estados Unidos debido a que comparten muy pocas similitudes con la cultura americana.

Por otro lado, la tesis de partida de muchas de las investigaciones apunta al posible aumento de los conflictos entre progenitores e hijos e hijas, derivado de las diferencias en la adaptación a la nueva cultura de adultos y menores, donde se presupone que los hijos e hijas presentan una mayor orientación hacia la cultura de acogida que sus progenitores. Una de las principales razones argumentadas es que los jóvenes inmigrantes adquieren la cultura del país de acogida de una forma más rápida que sus padres y madres, situación que ha sido considerada como potencialmente problemático para las relaciones entre ellos (Birman, 2006; Telzer, Yuen, Gonzales, \& Fuligni, 2016). El razonamiento seguido para apoyar esta idea es que los jóvenes están insertos en mayores contextos de socialización (usualmente se cita la escuela como el principal motor de aceleración de este resultado) que sus progenitores y por tanto están expuestos a mayores influencias de la cultura mayoritaria que ellos. Además, estos jóvenes están en pleno proceso de desarrollo, con lo cual ambos procesos (desarrollo y aculturación) se entremezclan, lo que genera una situación más compleja, mientras que sus padres y madres cuando emigran ya son adultos, usualmente han sido socializados dentro de la cultura de origen desde los primeros años, tienen los valores y prácticas más interiorizados, y aprenden el nuevo lenguaje con mayor dificultad que sus hijos e hijas (Fuligni, 2012). Sin embargo, 
es importante además tener en cuenta que no todos los adolescentes están más orientados hacia la cultura de acogida que hacia su cultura de origen o familiar; el problema es que en algunas investigaciones se infiere sin realizar ninguna medida de esta situación.

Por otro lado, algunos autores apuntan hacia la posibilidad de una menor presencia de conflictos en las familias procedentes de culturas colectivistas debido a que los valores de interdependencia son más destacados. De esta forma, en los grupos culturales en los que se valora más la autonomía, la capacidad de los adolescentes para tomar decisiones personales incluso si implica desafiar a los padres, es considerado un rasgo altamente deseable, mientras que en los grupos donde atender al beneficio de la colectividad es más importante que el ejercicio de la libre elección, esta situación sería vista de una forma negativa (Collins \& Steinberg, 2006). Sin embargo, esta situación también puede verse influenciada por el proceso de aculturación, dado que la adscripción a estos valores se verá modificada por la exposición a los valores de la cultura dominante. De este modo, cuando no existe consenso entre los progenitores y sus hijos e hijas sobre la relevancia de los valores, costumbres, reglas y prácticas, los conflictos en las familias inmigrantes pueden incrementarse (Berry \& Vedder, 2016). Como señalan Bulcroft, Carmody y Bulcroft (1996), "la potencial falta de congruencia entre los valores minoritarios y mayoritarios podría presentar un desafío para la legitimidad de los padres y podría minar la efectividad de los controles indirectos" (p. 867). De este modo, el proceder de una sociedad colectivista puede convertirse en una fuente de conflictos entre progenitores, hijos e hijas, en lugar de reducir su presencia, situación que se refleja en las preocupaciones de los padres y madres inmigrantes. Por ejemplo, en el estudio realizado en España por Micolta León (2007) se pone en relieve la preocupación de los padres y madres inmigrantes procedentes de Colombia en España acerca de la dificultad de transmitir los valores de su cultura de origen a sus hijos e hijas y que estos se identifiquen con los valores y pautas de la cultura española, y se señala presencia de conflictos con sus hijos e hijas debido a la confrontación entre su intención de educarlos de acuerdo con su marco cultural y la conducta de ellos de asumir las pautas del país receptor.

También Perreira, Chapman y Stein (2006), en sus entrevistas con padres y madres latinoamericanos emigrados a Estado Unidos, ponen de manifiesto la preocupación por la pérdida de la cultura de origen y la adquisición de las normas y conductas de la cultura mayoritaria por parte de sus hijos e hijas. Para concluir habría que señalar que en la revisión de Kwak (2003) sobre los estudios que se han centrado en las relaciones familiares intergeneracionales de inmigrantes y no inmigrantes, donde examina la hipótesis inicial de que habrá más descuerdo familiar y dificultades en las familias inmigrantes que en las no inmigrantes, una de las conclusiones que se presenta es que los adolescentes inmigrantes de sociedades colectivistas mantienen relaciones positivas con su familia, en parte retrasando la búsqueda de su autonomía.

De este modo, en este estudio pretendemos comprobar si los estilos de aculturación planteados por el modelo de Berry son identificables en los jóvenes latinoamericanos de primera generación que viven en España, y si su prevalencia es la encontrada de forma general. Posteriormente, pretendemos relacionar los perfiles de aculturación con los niveles de autonomía que los jóvenes tienen, así como con los conflictos que mantienen con sus progenitores y sus niveles de bienestar subjetivo. Concretamente, esperamos que los adolescentes que opten por el perfil de asimilación sean los que mayores conflictos y autonomía presenten, así como que los adolescentes en el perfil de marginación sean los que reporten un menor bienestar subjetivo. 


\section{Método}

\section{Participantes}

La muestra estuvo compuesta por alumnos de 21 centros de enseñanza secundaria de diversas localidades del sur de España. Se seleccionaron los centros en los que había una mayor proporción de alumnos inmigrantes, a partir de la información proporcionada por la Consejería de Educación. Como criterios de inclusión, tanto los jóvenes como sus dos progenitores debían haber nacido en el mismo país latinoamericano, y los jóvenes debían vivir al menos con uno de sus dos progenitores. La muestra final del estudio estuvo compuesta por 331 adolescentes procedentes de 15 países, principalmente de Ecuador (28.4\%), Bolivia (20.2\%) y Colombia (18.7\%). El 48.6\% fueron chicas y el $51.4 \%$ chicos, con edades comprendidas entre los 12 y los 18 años $(M=14.5$ años, $\mathrm{DE}=1.56)$. El tiempo que llevaban en España se situó en 4.51 años $(\mathrm{DE}=3)$.

\section{Instrumentos}

Para agrupar a los participantes en función de la estrategia de aculturación dominante, utilizamos tres instrumentos. En primer lugar, una versión modificada del Cuestionario de Identidad Étnica y Aculturación desarrollado por Bartolomé et al., (2000) y Espín, Marín, Rodríguez y Cabrera (1998). Este cuestionario de autoinforme está compuesto por dos subescalas (orientación hacia la cultura de origen y orientación hacia la cultura de acogida) compuestas cada una por 20 ítems que indagan acerca de una gran variedad de aspectos, desde conductas sociales y culturales observables, como el lenguaje, las relaciones de amistad, las funcionalidad del grupo étnico, los medios de comunicación y las tradiciones étnicas, hasta aspectos más internos, como las imágenes, ideas, actitudes y sentimientos. La escala de respuestas fue de tipo Likert de 3 puntos, donde puntuaciones mayores indicaban una mayor adherencia al ítem presentado. La consistencia interna de ambas subescalas fue adecuada ( $\alpha=0.88$ para la orientación hacia la cultura de origen y $\alpha=0.83$ para la orientación hacia la cultura de acogida). En segundo lugar tuvimos en cuenta la preferencia por el lugar de residencia, la escala de respuestas incluía 5 alternativas, de 1 (país de origen) a 5 (España). Por último, consideramos la identidad étnica tal y como es medida por la Escala de Identidad Étnica Multigrupo Revisada (Phinney \& Ong, 2007), concretamente la versión validada en España por Lara y Martinez-Molina (2016). Está compuesta por 6 ítems que miden la consecución de la identidad étnica, con una escala de respuesta de 5 puntos, de 1 (totalmente en desacuerdo) a 5 (totalmente de acuerdo). La consistencia interna fue adecuada, $\alpha=0.84$.

Para evaluar los conflictos que los jóvenes mantenían con sus progenitores así como su autonomía, se utilizó el Cuestionario de Relaciones Familiares desarrollado por Lara y Padilla (2010) compuesto por 26 ítems en los que se preguntaban dos cuestiones; quién toma la decisión en relación a ellos, de 1 (los progenitores deciden) a 5 (el adolescente decide) y cuántas veces habían discutido con sus progenitores en el último mes, de 1 (nunca) a 4 (más de 4 veces). La consistencia interna de ambas fue adecuada, $\alpha=0.84$ para autonomía y $\alpha=0.88$ para frecuencia de conflictos.

Por último, el bienestar subjetivo fue medido mediante la Escala de Satisfacción con la Vida, concretamente la traducción que Atienza, Pons, Balaguer, y García-Merita (2000) realizan de la original de Diener, Emmons, Larsen, y Griffin (1985). Está compuesta por 5 ítems que indagan sobre la evaluación global que las personas hacen de su vida, con una escala de respuesta de 1 (totalmente en desacuerdo) a 5 (totalmente de acuerdo). Presentó una consistencia interna adecuada, $\alpha=0.78$.

\section{Procedimiento}

Los cuestionarios fueron cumplimentados de forma anónima y colectiva por los participantes en los centros en los que estudiaban, en horario lectivo, previa autorización de los directores y obtención del consentimiento informado de los 
progenitores. Los cuestionarios fueron aplicados por la misma investigadora en todos los centros.

Análisis de datos

La agrupación de los jóvenes en función de las estrategias de aculturación adoptadas se llevó a cabo mediante un análisis de conglomerados (método K-medias), utilizando las puntuaciones tipificadas ofrecidas por las dos subescalas del cuestionario de Identidad étnica y de Aculturación (orientación hacia la cultura de origen y orientación hacia la cultura de acogida), la Escala de Identidad Étnica Multigrupo Revisada y la preferencia por el lugar de residencia. Con los perfiles encontrados se llevaron a cabo análisis de la varianza para comprobar si existían diferencias en los niveles de autonomía de los adolescentes y los conflictos que mantenían con sus progenitores, así como en la satisfacción vital. Sé utilizó la prueba de comparaciones múltiples a posteriori de Scheffé para analizar entre qué perfiles se producían las diferencias significativas encontradas a nivel global mediante los análisis de la varianza. Todos los análisis estadísticos se llevaron a cabo utilizando el programa IBM SPSS Statistics 21.

\section{Resultados}

En la Figura 1 se representan las puntuaciones tipificas medias obtenidas en las variables introducidas para calcular los conglomerados. El primer conglomerado se identificó claramente por presentar un perfil de separación; estuvo conformado por 100 adolescentes con puntuaciones por encima de la media en orientación hacia la cultura de origen e identidad étnica, y por debajo en orientación hacia la cultura de acogida y preferencia por vivir en España. El segundo conglomerado representó el perfil de integración, que agrupaba a 113 jóvenes que presentaron puntuaciones por encima de la media en todas las variables introducidas. El tercer conglomerado quedó conformado por 55 adolescentes y representó el perfil de asimilación, pues mostraba el patrón opuesto al perfil de separación. El último conglomerado, que agrupó a 63 adolescentes, fue denominado perfil de marginación debido a que presentó puntuaciones por debajo de la media en todas las variables introducidas.

\section{Figura 1}

Perfiles de aculturación

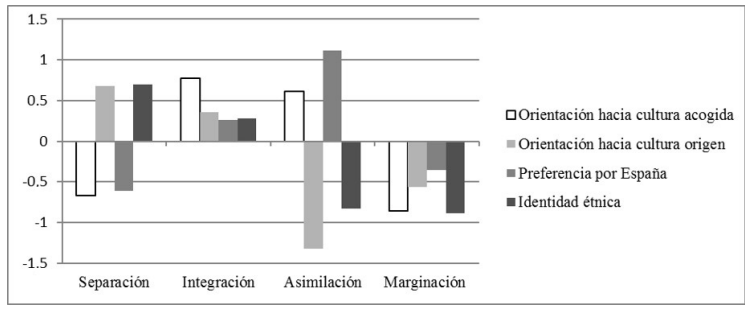

Fuente: elaboración propia.

Los resultados de las comparaciones entre los perfiles encontrados en las puntuaciones de las variables familiares (autonomía conductual y frecuencia de conflictos adolescentesprogenitores) y de satisfacción con la vida se muestran en la Tabla 1. No se encontraron diferencias estadísticamente significativas ni en los conflictos que mantenían con sus progenitores ni en la autonomía conductual en función de los perfiles de aculturación. Sin embargo, la satisfacción con la vida de los adolescentes sí mostró variaciones en función de los conglomerados de pertenencia. Las pruebas de comparaciones múltiples a posteriori mostraron que los adolescentes que optaron por la marginación presentaron menores puntuaciones que el resto de perfiles.

\section{TABLA 1}

Diferencias en el estilo de aculturación en las variables familiares y la satisfacción vital

\begin{tabular}{lllllll}
\hline & Separación & Integración & Asimilación & Marginación & \\
& $M(\mathrm{DT})$ & $M(\mathrm{DT})$ & $M(\mathrm{DT})$ & $M(\mathrm{DT})$ & $F$ & $p$ \\
\hline Conflictos & $1.43(0.42)$ & $1.44(0.46)$ & $1.47(0.35)$ & $1.5(0.47)$ & 0.384 & 0.764 \\
Autonomía & $3.33(0.54)$ & $3.31(0.55)$ & $3.35(0.62)$ & $3.45(0.51)$ & 0.933 & 0.425 \\
Satisfacción & $3.78(0.74)$ & $3.9(0.85)$ & $3.65(0.82)$ & $3.18(0.93)$ & 10.63 & 0 \\
\hline
\end{tabular}

Fuente: elaboración propia. 


\section{Discusión}

Los perfiles encontrados son similares a los hallados en otras investigaciones que han tratado de agrupar a los inmigrantes en función de diversas variables relacionadas con el proceso de aculturación (e.g., Berry et al., 2006; Sobral et al., 2010). La identificación de estos perfiles permite estudiar las variables tenidas en cuenta de una forma más comprehensiva, tomando en consideración todos los aspectos estudiados a la vez. La integración se presenta como la opción predominante, coincidente con las investigaciones previas (Berry et al., 2006; Briones, 2010; Sobral et al., 2010; Telzer et al., 2016). Esta situación se percibe como positiva, dado que la integración es la estrategia que ha demostrado tener mejores resultados para la adaptación de los adolescentes inmigrantes. De esta forma, los jóvenes inmigrantes de origen latinoamericano en España se desenvuelven de forma adecuada en ambos contextos culturales, pueden mantener los valores, costumbres y creencias de su cultura de origen a la vez que aprenden y valoran los de la sociedad en la que se integran. A su vez, esto les permite adquirir las competencias culturales necesarias para desenvolverse en la sociedad de acogida y les posibilita desarrollar una identidad étnica adecuada.

Sin embargo, aunque la situación de adaptación de los jóvenes inmigrantes en España podría definirse como positiva, es necesario señalar otra serie de resultados que apuntan hacia la posible aparición de problemas. En primer lugar, el porcentaje de adolescentes que se decantaron por el perfil de separación fue muy similar al de integración. Estos jóvenes están orientados hacia la cultura del país en el que nacieron y no se identifican, o se identifican poco, con la nueva cultura. Llevar a cabo esta estrategia de aculturación puede deberse a una magnificación de su cultura cuando se sienten rechazados por la sociedad en la que se integran. Algunos autores han apuntado, por ejemplo, a que el rechazo social que algunos latinos sienten de la sociedad de acogida provoca que se orienten hacia la cultura de origen (Delpino
Gouicochea, 2007). Esto puede dar lugar a la formación de bandas de carácter violento con compatriotas, donde buscan el afecto, la protección y la creación de una identidad que no encuentran en la sociedad de acogida. Por ejemplo, Zlobina, Basabe y Páez (2004), quienes también señalan como causa de la aparición de esta estrategia el rechazo de la sociedad de acogida, encuentran que esta es la estrategia que se asocia con mayores problemas emocionales. Además, sobre esta situación es posible que esté influyendo la creación de barrios y escuelas con elevada concentración de inmigrantes que puede estar dando lugar a una menor orientación y deseo de integrarse en la sociedad de acogida. Sin embargo, a pesar de que la existencia de este porcentaje de jóvenes que llevan a cabo la estrategia de separación puede ser interpretado como un aspecto negativo que tendremos que tener presente a la hora de las intervenciones, también es necesario señalar que los inmigrantes que participaron en el estudio pueden ser considerados de forma global recientes, dado que el tiempo medio de estancia en España se situó en torno a los cuatro años y medio. De esta forma, la estrategia de separación es usual en personas que llevan muy poco tiempo en la cultura de acogida (Bartolomé et al., 2000). Por último, la marginación no fue la última opción, contrario a lo que se esperaba. Esta situación debe tenerse en cuenta, dado que de forma sistemática se ha encontrado que son los que pueden tener peores resultados de adaptación, puesto que deja a los jóvenes en un estado de vacío, con un sentimiento de que no pertenecen a ninguna cultura; resultado que confirmamos en relación a la satisfacción con la vida, pues son el grupo que presenta menores puntuaciones y estas son significativamente inferiores a las obtenidas por el resto de grupos.

En relación a la autonomía de los jóvenes inmigrantes, así como los conflictos que mantienen con sus progenitores, es destacable que las variables relacionadas con la aculturación no ejerzan ninguna influencia. En primer lugar, esperábamos que una mayor orientación hacia la cultura de acogida que hacia la de origen (perfil de asimilación) estuviese relacionada con 
una mayor autonomía, basándonos en la idea de que en la sociedad española es más valorada que en las de origen de los inmigrantes. En segundo, también esperábamos encontrar un aumento de los conflictos en aquellos perfiles más orientados hacia la cultura de acogida, concretamente en el de asimilación, partiendo de que la aculturación más rápida de los hijos e hijas era una de las circunstancias que se habían señalado como influyentes sobre un aumento de los conflictos con los progenitores (García-Coll, 2005; Telzer et al., 2016). De esta forma, una mayor orientación hacia la sociedad española debería de alguna manera implicar un aumento en la autonomía y los conflictos. Sin embargo, otras explicaciones alternativas también son posibles al hecho de no confirmarse estas hipótesis. Por ejemplo, puede que una orientación mayor o menor por sí misma no dé lugar a una mayor autonomía, sino que dependerá de cómo se gestione esta influencia dentro de la estructura familiar. Nos hemos basado en la autonomía que los adolescentes realmente tienen, por lo que puede que la orientación hacia la cultura de acogida sí hubiese dado lugar a un aumento del deseo por alcanzarla. En relación a lo anterior, puede que los padres y madres, al encontrarse en un nuevo contexto en el que consideran que estos jóvenes deberían tomar una mayor cantidad de decisiones para poder adaptarse y convertirse en miembros autónomos en esta nueva sociedad en la que van a vivir, realmente les concedan dicha autonomía, independientemente de la orientación de los menores. Además, en relación concretamente a la frecuencia de conflictos, una posible explicación complementaria es que estos perfiles de aculturación de los adolescentes sean congruentes con los de sus progenitores, situación que sería interesante analizar en futuras investigaciones.

Por último, queremos destacar que una explicación plausible para los resultados encontrados en nuestro contexto cultural se deba a características propias de las similitudes con las culturas de origen de los inmigrantes. Es posible simplemente que la tesis de partida no sea aplicable en este contexto concreto, dado que la autonomía puede no ser más valorada en la cultura española que en las culturas de origen de los jóvenes inmigrantes. Las investigaciones que planteaban este tipo de relaciones habían sido principalmente llevadas a cabo en contextos que consideramos más individualistas, como puede ser el caso de los Estados Unidos, en donde además se presentaría mayor diferencia inicial entre las culturas. Por tanto, esta investigación constituye un nuevo aporte al estudio de las relaciones familiares, poniendo de relieve la importancia de la influencia de los contextos culturales.

\section{Agradecimientos}

A María Luisa Padilla, profesora, directora, compañera y amiga. Gracias por todo, más allá de lo académico, gracias por ser una constante en mi vida, siempre impulsándome a conseguir mis metas. Estés donde estés, espero de alguna forma sepas que lo conseguimos, porque esto, como la mayoría de mis logros, también es tuyo.

\section{Referencias}

Atienza, F. L., Pons, D., Balaguer I., \& García-Merita, M. L. (2000). Propiedades psicométricas de la Escala de Satisfacción con la Vida en adolescentes. Psicothema, 12(2), 331-336.

Bartolomé Pina, M., Cabrera Rodríguez, F., Espín López, J. V., Campo del Sorribas, J., Marín Gracia, M. A., Rodríguez Lajo, M., et al. (2000). La construcción de la identidad en contextos multiculturales. Madrid: Ministerio de Educación, Cultura y Deporte. Secretaría General de Educación y Formación Profesional (CIDE). Recuperado de: http://www.dored in.mec.es/documentos/008200120101.pdf .

Berry, J. W. (1997). Immigration, acculturation, and adaptation. Applied Psychology: International Review, 46(1), 5-34.

Berry, J. W., \& Vedder, P. (2016). Adaptation of immigrant children, adolescents and their families. En U. P. Gielen y J. L. Roopnarine (Eds.), Childhood and adolescence: Cross- 
cultural perspectives and applications (2nd ed., pp. 321-346). Santa Barbara: Praeger.

Berry, J. W., Phinney, J. S., Sam, D. L., \& Vedder, P. (2006). Immigrant youth in cultural transition: Acculturation, identity and adaptation across national contexts. Mahwah, NJ: Lawrence Erlbaum.

Birman, D. (2006). Measurement of the "acculturation gap" in immigrant families and implications for parent-child relationships. En M. H. Bronstein \& L. R. Cote (Eds.), Acculturation and parent-child relationships: Measurement and development (pp. 113-134). Mahwah, NJ: Lawrence Erlbaum.

Briones, E. (2010). La aculturación de los adolescentes inmigrantes en España: Aproximación teórica y empírica a su identidad cultural y adaptación psicosocial. Salamanca: Ediciones Universidad Salamanca.

Bulcroft, R. A., Carmody, D. C., \& Bulcroft, K. A. (1996). Patterns of parental independence giving to adolescents: Variations by race, age, and gender of child. Journal of Marriage and the Family, 58(4), 866-883. doi: http://dx.doi.org/10.2307/35 3976

Choi, Y., He, M., \& Barachi, T. W. (2008). Intergenerational cultural dissonance, parent-child conflict and bonding, and youth problem behaviors among Vietnamese and Cambodian immigrant families. Journal of Youth Adolescence, 37 (1), 85-96. doi: http://dx.doi.org/10.1007/s1096 4-007-9217-z

Coatsworth, J. D., Maldonado-Molina, M., Pantin, H., \& Szapocznik, J. (2005). A person-centered and ecological investigation of acculturation strategies in Hispanic immigrant youth. Journal of Community Psychology, 33(2), 157-174.

Collins, W. A., \& Steinberg, L. (2006). Adolescent development in interpersonal context. En Damon y R. Lerner (Series Eds) y N. Eisenberg (Vol. Ed.), Handbook of Child Psychology: Vol. 3. Social, emotional, and personality development (pp. 1003-1067). Nueva York, NY: John Wiley \& Sons.
David, E. J. R., Okazaki, S., \& Saw, A. (2009). Bicultural self-efficacy among college students: Initial scale development and mental health correlates. Journal of Counseling Psychology, 56(2), 211-226.

Delpino Gouicochea, M. A. (2007). La inserción de los adolescentes latinoamericanos en España: Algunas claves. Madrid: Ministerio de Trabajo y Asuntos Sociales.

Diener, E., Emmons, R. A., Larsen, R. J., \& Griffin, S. (1985). The satisfaction with life scale. Journal of Personality Assessment, 49(1), 71-75.

Espín, J. V., Marín, M. A., Rodríguez, M., \& Cabrera, F. (1998). Elaboración de un cuestionario para medir la identidad étnica y la aculturación en la adolescencia. Revista de Educación, 315, 227-249.

Fuligni, A. J. (2012). Gaps, conflicts, and arguments between adolescents and their parents. New Directions for Child and Adolescent Development, 135, 105-110.

García-Coll, C. (2005). ¿Cuándo se convierte la cultura en un factor de riesgo contextual?. En E.L. Ezpeleta (Ed.), Factores de riesgo en psicopatología del desarrollo (pp. 237-255). Barcelona: Masson.

Horrocks, J. E. (1962). The psychology of Adolescence, behaviour and development (2a ed.). Boston, M.A: Houghton Mifflin.

Kashima, E. S., \& Abu-Rayya, H. M. (2014). Longitudinal associations of cultural distance with psychological wellbeing among Australian immigrants from 49 Countries. Journal of Cross-Cultural Psychology, 45(4), 587-600. doi: http://dx.d oi.org/10.1177/0022022113519857

Kwak, K. (2003). Adolescent and their parents: A review of intergenerational family relations for immigrant and non-immigrant families. Human Development, 46(2-3), 115-136.

Lara, L., \& Martínez-Molina, A. (2016). Validación de la Escala de Identidad Étnica Multigrupo-Revisada en adolescentes inmigrantes y autóctonos residentes en España. Revista Latinoamericana de Ciencias Sociales, Niñez y Juventud, 14(1), 
591-601. http://dx.doi.org/10.11600/16927 15x.14140110515

Lara, L., \& Padilla, M. L. (2010). Cuestionario para el estudio de los conflictos entre padres e hijos inmigrantes y autóctonos. En E. Soriano, A. Gónzalez, y R. M. Zapata (Eds.), Retos Internacionales ante la Interculturalidad (pp. 76-81). Almería, España: Editorial Universidad de Almería.

Matsunaga, M., Hecht, M. L., Elek, E., \& Ndiaye, K. (2010). Ethnic identity development and acculturation: A longitudinal analysis of Mexican-heritage youth in the southwest Unites States. Journal of Cross-Cultural Psychology, 41 (1), 410-427. doi: http://dx.d oi.org/10.1177/0022022109359689

Micolta León, A. (2007). Inmigrantes colombianos en España: Experiencia parental e inmigración. Revista Latinoamericana de Ciencias Sociales, Niñez y Juventud, 5(1), 1-25.

Nguyen, A-M. D., \& Benet-Martínez, V. (2013). Biculturalism and adjustment: A meta-analysis. Journal of Cross-Cultural Psychology, 44(1), 122-159. doi: http://dx.d oi.org/10.1177/0022022111435097

Perreira, K. M., Chapman, M. V., \& Stein, G. L. (2006). Becoming an American parent: Overcoming challenges and finding strength in a new immigrant Latino community. Journal of Family Issues, 27, 1383-1414. doi: http://dx.doi.org/10.1177/ $0192513 X 06290041$

Phinney, J. S., \& Ong, A. D. (2007). Conceptualization and measurement of ethnic identity: Current status and future directions. Journal of Counseling Psychology, 54(3), 271-281.

Rudmin, F. W. (2003). Critical history of the acculturation psychology of assimilation, separation, integration and marginalization. Review of General Psychology, 7(1), 3-37.

Sam, D. L., \& Oppedal, B. (2003). Acculturation as a Developmental Pathway. Online Readings in Psychology and Culture, 8(1). htt p://dx.doi.org/10.9707/2307-0919.1072
Schwartz, S. J., Unger, J. B., Zamboanga, Byron L., \& Szapocznik, J. (2010). Rethinking the concept of acculturation: Implications for theory and research. American Psychologist, 65 (4), 237-251. doi: http://dx.doi.org/10.10 37/a0019330

Shafaei, A., Razak, N. A., \& Nejati, M. (2016). Integrating two cultures successfully: Factors influencing acculturation attitude of international postgraduate students in Malaysia. Journal of Research in International Education, 22, 1-18. doi: http://dx.doi.org/1 $0.1177 / 1475240916653566$

Sobral, J., Gómez-Fraguela, J. A., Luengo, A., Romero, E., \& Villar, P. (2010). Adolescentes latinoamericanos, aculturación y conducta antisocial. Psicothema, 22 (3), 410-415.

Sobral, J., Gómez-Fraguela, J. A., Romero, E., Luengo, A., \& Villar, P. (2012). Riesgo y protección de desviación social en adolescentes inmigrantes: Personalidad, familia y aculturación. Anales de Psicología, 28(3), 664-674.

Telzer, E. H., Yuen, C., Gonzales, N., \& Fuligni, A. J. (2016). Filling gaps in the acculturation gap-distress model: Heritage cultural maintenance and adjustment in Mexican-American families. Journal of Youth and Adolescence, 45(7), 1412-1426. doi: http://dx.doi.org/10.1007/s10964-0150408-8

Trueba, H. T. (2001). Múltiples identidades étnicas, raciales y culturales en acción: Desde la marginalidad hasta el nuevo capital cultural en la sociedad moderna. En E. Soriano (Coord.). Identidad cultural y ciudadanía: Su contexto educativo (pp. 17-44). Madrid: La Muralla, S.A.

Vedder, P., Van de Vijver, F. R. S., \& Liebkind, K. (2006). Predicting immigrant youths' adaptation across countries and ethnocultural groups. En J. W. Berry, J. S. Phinney, D. L. Sam y P. Vedder (Eds.), Immigrant youth in cultural transition: Acculturation, identity, and adaptation across national contexts (pp. 143-164). Mahwah, NJ: Lawrence Erlbaum. 
Ward, C., \& Geeraert, N. (2016). Advancing acculturation theory and research: The acculturation process in its ecological context. Current Opinion in Psychology, 8, 98-104. http://dx.doi.org/10.1016/j.copsyc. 2015.09.021

Zlobina, A., Basabe, N., \& Páez, D. (2004). Adaptación de los inmigrantes extranjeros en España: Superando el choque cultural. Migraciones, 5, 43-84.

\section{Notas}

* Artículo de investigación. 